\title{
Formulation of Mathematical Model for TB Transmission in Zoonotic Areas with Existence of Endemic Equilibrium
}

\author{
Thomas Mwenyeheri ${ }^{*}$, Nyimvua Shaban², Doroth Hove-Msekwa ${ }^{3}$, \\ Symon Bilesi Chibaya ${ }^{4}$, Esther Ngadaya ${ }^{1}$, Sayoki Mfinanga ${ }^{1}$ \\ ${ }^{1}$ National Institute for Medical Research, Muhimbili Medical Research Centre, Dar es salaam, Tanzania \\ ${ }^{2}$ Department of Mathematics, University of Dar es salaam, Dar es salaam, Tanzania \\ ${ }^{3}$ Department of Applied Mathematics, National University of Science and Technology, Bulawayo, Zimbabwe \\ ${ }^{4}$ Basics Sciences Department, Malawi University of Science and Technology, Thyolo, Malawi \\ Email: ${ }^{*}$ tmwinyeheri@yahoo.com
}

Received 11 June 2014; revised 15 July 2014; accepted 1 August 2014

Copyright (C) 2014 by authors and Scientific Research Publishing Inc.

This work is licensed under the Creative Commons Attribution International License (CC BY).

http://creativecommons.org/licenses/by/4.0/

c) (i) Open Access

\section{Abstract}

A mathematical model incorporating animal TB and Human TB transmission is formulated and analysed in order to determine the role of animal TB and human TB in the overall TB transmission and also, to determine the parameters which govern the transmission of the TB disease. The model has five classes namely susceptible, exposed animal TB, and exposed human TB, infectious and recovery. The model assumed that there are two classes for infected individuals, those who acquired TB through animal and those who acquire TB from human. Qualitative results show that the model has the disease-free equilibrium and at least one endemic equilibrium that is locally asymptotically stable. The study includes numerical simulations as a way of supporting the analytical results. Graphical results indicate that animal TB has major contribution on overall TB transmission and the TB transmission can be reduced by ensuring intervention to both Animal TB and Human TB. Furthermore the equations indicate that there is at least one endemic equilibrium which translates that $t$ animal and human have the contribution on TB transmission. This shows that both animals and humans together with fast progressors have contribution on TB transmissions.

\section{Keywords}

TB, Mathematical Modeling, Animal, Human, Diseases Free-Equilibrium, Endemic Equilibrium

\footnotetext{
${ }^{*}$ Corresponding author.
}

How to cite this paper: Mwenyeheri, T., et al. (2014) Formulation of Mathematical Model for TB Transmission in Zoonotic Areas with Existence of Endemic Equilibrium. Journal of Tuberculosis Research, 2, 132-143. 


\section{Introduction}

\subsection{General Introduction}

Tuberculosis is the deadly diseases in developing countries, globally it causes many death than other diseases including Malaria, Schistosomiasis, HIV, Typhoid fever [1].

The source of infection of Tuberculosis (TB) can be from Human, Animal or environmental. That is Human to Human TB is caused by bacteria called Mycobacterium tuberculosissa. The bacteria can be transmitted through the air from one person to another. Zoonotic tuberculosis is caused by bacteria called Mycobacterium bovis (M. Bovis) and is transmitted from animal to human. Zoonotic tuberculosis is spread to a person by contacting or consuming product from infected animal. Environmental TB is caused by the surroundings in which water and soil can be a source of infection.

The bacteria can affect any part of the body, but they usually attack the lungs [pulmonary TB (PTB)], and it can also affect any other part of the body [extra-pulmonary TB (EPTB)]. EPTB with no evidence of pulmonary involvement accounted for $21.1 \%$ of those with TB in 2005, which represents an increase from 16\% in 1992 [2].

Human TB is spread by air in which the bacteria are put into the air when a person with TB disease of the lungs or throat coughs or sneezes.

Zoonotic tuberculosis was once a major problem in developed countries but following eradication programs involving test and slaughter policy and milk pasteurization [3], the incidence has drastically reduced [4]. However, the infection currently poses a major concern in the human population in developing countries, as humans and animals share the same microenvironment and dwelling premises, especially in rural areas [5].

The World Health Organization (WHO) estimates that over 2 billion people (one third of the world population) is infected with tuberculosis [6]. The same fact sheet stated that in 2008, globally, there were 9.37 million new cases of TB, with the African region and the Southeast Asian region (SEAR) having a share of $30 \%$ and $34 \%$, respectively. However, the estimated incidence rate in Sub-Saharan Africa is nearly twice that in the SEAR with over 350 cases per 100,000 individuals of the human population. In the same year 2008, an estimated 1.3 million people died from TB. The highest number of deaths was in SEAR, while the highest mortality per capita was in the African region.

Transmission of $M$. bovis can occur between animals, from animals to humans and vice versa and rarely, between humans. M. bovis infection is transmissible from cattle to humans directly by erogenous route [3] and through direct contact with material contaminated with nose and mouth secretions from an infected herd of cattle ([7] cited on [8]). Also, the existing eating culture (eating of raw meat and drinking of raw milk), the very common close contact of animals with humans (most common in rural areas), inadequate meat inspection and the prevailing low standard of hygienic practices are potential risk factors that favours the spreading of zoonotic tuberculosis [6].

\subsection{Statement of the Problem}

Zoonotic transmission of tuberculosis to human populations is well recognized and is estimated to account for more than $10 \%$ of all forms of TB infections in vulnerable communities in Tanzania [9]. Infected animals are a major source of TB, thus indirectly subjecting livestock-keeping families to long-term poor health and poverty. The extent of zoonotic transmission of TB in urban populations in Tanzania and how the rural-pastoralist transmission affects the general transmission patterns of TB has not been studied in the past. [10] has studied the transmission of TB on contact transmission but ignored contact of animal and human which lead infected animals to transmit TB to human. Therefore this study uses [10] model to incorporate animal to human transmission in order to have a model which indicate the transmission of animal TB.

\subsection{Research Objective}

The main objective of this study was to determine TB transmissions rate caused by contact and animal to human transmission in zoonotic heterogeneous mixing population. The specific objectives of this study were:

1) To formulate and analyze a mathematical model for TB transmission in zoonotic heterogeneous mixing population.

2) To determine the behavior of parameters which govern the transmission of zoonotic TB. 


\subsection{Significance of the Study}

This study will give a better understanding of the zoonotic TB using mathematical models. The established prevalence and transmission patterns and dynamics of zoonotic TB from this study will build a strong and functional collaboration between the National Tuberculosis and Leprosy Program (NTLP) and the veterinary and Wildlife departments for control of zoonotic transmission of TB for the increased knowledge about geographical distribution of zoonotic TB diseases in selected rural and urban populations.

\section{The Tuberculosis Model for Transimission of Zoonotic Tuberculosis}

\subsection{Introduction}

In this chapter we are going to formulate and analyze the model. The model will include fast progressors to active TB, animal TB and Human TB disregarding environmental TB. The model will incorporate five classes of individuals which are susceptible, infected by animal TB, infected by human TB, infectious and recovered. The study will carry analysis on examining the positivity of the model, finding the disease free and endemic equilibrium points.

\subsection{Model Formulation}

The mathematical model incorporates animal and human TB to determine their contribution and transmission rate to TB dynamics. Population is divided into five classes as follows: susceptible $S(t)$ individuals who are free from TB infection but are at risk, exposed individuals have been divided in two classes as exposed to human TB $E_{H}(t)$ (came in contact with human TB) and those exposed to animal TB $E_{A}(t)$ (came in contact with animal or consumed infected animal products like drinking raw milk, eating raw meat), Infected $I(t)$ individuals and Recovered $R(t)$ individuals. The total human population is given by $N=S+E_{H}+E_{A}+I+R$.

We assume that, new recruits enter the susceptible class at a constant rate, $\Pi$. The susceptible population is diminished by natural death at a constant rate $\mu$. A proportion $q$ of susceptible individuals is exposed to human TB and $(1-q)$ is exposed to animal TB. A proportion $p$ of susceptible who come in contact with infectious individuals become infectious, that is, develop active TB and $(1-p)$ are infected but not infectious, that is, become exposed individuals or have latent TB. Therefore individuals are exposed to human TB at a rate of $q(1-p) S(t)$ and to animal TB at a rate of $(1-q)(1-p) S(t)$. The rates $\tau$ and $\Upsilon$ indicate the progression rates from exposed TB class in human class and animal class to infectious class respectively. Therefore there are $\tau E_{H}$ and $\Upsilon E_{A}$ new infectious individuals per unit of time from human and animal classes respectively.

Total per capita removal rate is defined by $\gamma$ and it is a composition of mortality natural death rate $\mu$, TB death rate and recovery rate $r$. This implies that $\gamma=\mu+d+r$. The force of infection is $\beta=\alpha I / N$, where $\alpha$ is the transmission rate (Table 1 ).

Taking into account the above considerations, we have the following schematic flow diagram (Figure 1).

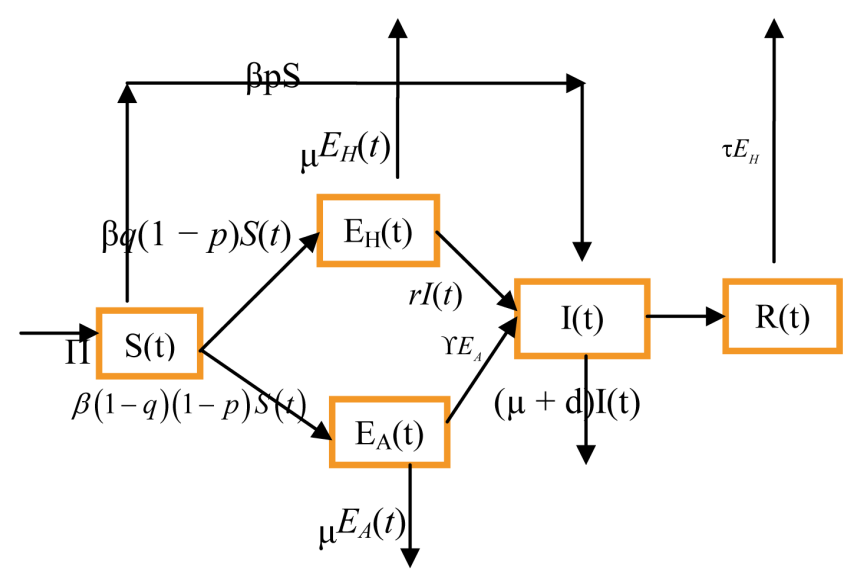

Figure 1. Model flow chart. 
Table 1. Model variables and parameters.

\begin{tabular}{|c|c|}
\hline Variables/Parameters & Definitions \\
\hline$S(t)$ & Number of susceptible Individuals time, $t$ \\
\hline$E_{H}(t)$ & Number of latently human TB infected individual at time, $t$ \\
\hline$E_{A}(t)$ & Number of latently animal TB infected at time, $t$ \\
\hline$I(t)$ & Number of infectious individual at time, $t$ \\
\hline$R(t)$ & Number of infectious individual in treatment at time, $t$ \\
\hline$\mu$ & Natural death \\
\hline$P$ & Fraction of fast progressors to the active disease \\
\hline $1-p$ & Proportion of susceptible population to latent TB (Animal and Human) \\
\hline$Q$ & Proportion of susceptible population to latent Human TB \\
\hline $1-q$ & Proportion of susceptible population to latent Animal TB \\
\hline$q(1-p)$ & $\begin{array}{l}\text { Proportion of susceptible class to human TB (Human Latent TB) after adjustment with susceptible class to } \\
\text { infected class }(1-p)\end{array}$ \\
\hline$(1-q)(1-p)$ & $\begin{array}{l}\text { Proportion of susceptible class to exposed animal TB (Animal Latent TB) after adjustment with } \\
\text { susceptible class to infected class }\end{array}$ \\
\hline$\tau$ & Indicate the progression rate from exposed human TB class to infectious class \\
\hline$\Upsilon$ & Indicate the progression rate from exposed animal TB class to infectious class \\
\hline$R$ & Recovery rate from infectious class \\
\hline$D$ & Per capita mortality rate due to TB disease \\
\hline$\gamma=\mu+d+r$ & Total per capita remove rate from infectious population \\
\hline A & Transmission rate for the susceptible class to the infective class \\
\hline$B$ & Force of infection and alpha is the transmission rate for the susceptible class to the infective class \\
\hline
\end{tabular}

\subsection{Model Assumption}

- The Model will not consider environmental tuberculosis.

- Individuals are equally likely to be infected by the infectious individual in case of contact.

From the descriptions, assumptions and biological flow chart in Figure 1, we obtained the following model equations.

$$
\begin{aligned}
& \frac{\mathrm{d} S}{\mathrm{~d} t}=\Pi-(\beta+\mu) S, \\
& \frac{\mathrm{d} E_{H}}{\mathrm{~d} t}=\beta q(1-p) S-(\mu+\tau) E_{H}, \\
& \frac{\mathrm{d} E_{A}}{\mathrm{~d} t}=\beta(1-q)(1-p) S-(\mu+\Upsilon) E_{A}, \\
& \frac{\mathrm{d} I}{\mathrm{~d} t}=\tau E_{H}+\Upsilon E_{A}+\beta p S-(\mu+d+r) I, \\
& \frac{\mathrm{d} R}{\mathrm{~d} t}=r I-\mu R .
\end{aligned}
$$

where $\beta=\frac{\alpha I}{N}$ and $N=S+E_{H}+E_{A}+I+R$.

\section{Basic Properties of the Model}

In this section we investigate the feasibility and positivity of the solution. The feasibility of the model describes the region in which the solution of the system (3.1) are biological meaningful. Positivity of solution describes non-negative solution of system (3.1). 


\subsection{Feasible Solution}

The feasible solution which is a positively invariant set of the model is given by $\Psi=\left(\left(S, E_{H}, E_{A}, I, R\right) \in R_{+}^{5}: N \leq \frac{\Pi}{\mu}\right)$, at disease free equilibrium, from the function of

$N=S+E_{H}+E_{A}+I+R$ therefore the change of $N$ will lead to changes to all variables in the population, then we will obtain

$$
\frac{\mathrm{d} N}{\mathrm{~d} t}=\frac{\mathrm{d} S}{\mathrm{~d} t}+\frac{\mathrm{d} E_{A}}{\mathrm{~d} t}+\frac{\mathrm{d} E_{H}}{\mathrm{~d} t}+\frac{\mathrm{d} I}{\mathrm{~d} t}+\frac{\mathrm{d} T}{\mathrm{~d} t}
$$

which leads to

$$
\frac{\mathrm{d} N}{\mathrm{~d} t}=\Pi-\left(S+E_{H}+E_{A}+I+R\right) \mu-I d
$$

Therefore in the absence of diseases $(d=0)$ and $N=S+E_{H}+E_{A}+I+R$ we obtain

$$
\frac{\mathrm{d} N}{\mathrm{~d} t}=\prod-N \mu
$$

From Equation (3.4) we observe that,

$$
\frac{\mathrm{d} N}{\mathrm{~d} t}<0 \quad \text { if } \quad N>\frac{\Pi}{\mu}
$$

By using Birkhoff and Rota’s theorem (1989) on differential inequalities we obtain,

$$
N(t) \leq N(0) \mathrm{e}^{\mu t}+\frac{\Pi}{\mu}\left(1-\mathrm{e}^{-\mu t}\right)
$$

Therefore as $t \rightarrow \infty, N \leq \frac{\Pi}{\mu}$. Therefore $\Psi$ is positively invariant.

\subsection{Positivity of Solutions}

In this section we elaborate the non-negativity of the solution of the system (3.1), by considering the lemma below.

Lemma 1: Let initial value of the system (3.1) be $\left(S, E_{H}, E_{A}, I, T, R\right)(0)$. Then the solution set of the system (3.1) $\left(S, E_{H}, E_{A}, I, T, R\right)(t)$ is positive for all $t>0$.

Proof: Consider the first equation of system (3.1),

$$
\frac{\mathrm{d} S}{\mathrm{~d} t}=\Pi-(\beta+\mu) S \geq-(\beta+\mu) S
$$

Integrating Equation (3.7) with respect to time $t$, we obtain

$S(t) \geq S(0) \mathrm{e}^{-\int(\beta+\mu) t} \geq 0$ which is positive as explained in above lemma.

From Equation (2) of system (3.1)

$$
\frac{\mathrm{d} E_{H}}{\mathrm{~d} t}=\beta q(1-p) S-\mu E_{H}-\tau E_{H} \geq-(\mu+\tau) E_{H}
$$

Integrating Equation (3.8) with respect to $E_{H}$ we obtain,

$E_{H}(t) \geq E_{H}(0) \mathrm{e}^{-(\mu+\tau) t} \geq 0$, showing that the variable $E_{H}$ is positive.

Consider fifth equation of the system (3.1)

$$
\frac{\mathrm{d} R}{\mathrm{~d} t}=r I-\mu R>-\mu R
$$

By integration, we obtain 
$R(t) \geq R(0) \mathrm{e}^{-\mu t} \geq 0$. This indicates also that $R(t)$ is positive.

Consider equation 4 of system (3.1).

$$
\frac{\mathrm{d} I}{\mathrm{~d} t}=\tau E_{H}+\Upsilon E_{A}+\beta p S-(\mu+d+r) I>-(\mu+d+r) I
$$

Also integrating the fourth Equation (3.10) yields,

$I(t) \geq I(0) \mathrm{e}^{-(\mu+d+r) t}>0$ meaning that $I(t)$ is positive.

Therefore we have shown that all variables are positive for all $t>0$.

\subsection{Existence of Steady States of the System}

The equilibrium states are obtained by equating equations of the system (3.1) to zero that means $\frac{\mathrm{d} S}{\mathrm{~d} t}=\frac{\mathrm{d} E_{H}}{\mathrm{~d} t}=\frac{\mathrm{d} E_{\mathrm{A}}}{\mathrm{d} t}=\frac{\mathrm{d} I}{\mathrm{~d} t}=\frac{\mathrm{d} R}{\mathrm{~d} t}=0$.

So the system (3.1), becomes

$$
\begin{aligned}
& 0=\Pi-(\beta+\mu) S, \\
& 0=\beta q(1-p) S-(\mu+\tau) E_{H}, \\
& 0=\beta(1-q)(1-p) S-(\mu+\Upsilon) E_{A}, \\
& 0=\tau E_{H}+\Upsilon E_{A}+\beta p S-(\mu+d+r) I, \\
& 0=r I-\mu R .
\end{aligned}
$$

The disease-free equilibrium point is the situation where there is no infection in the population that means, $I=E_{H}=E_{A}=0$ in the system (3.1).

After solving the free-equilibrium point of the system (3.1), the first equation of system (3.1) gave us $S=\frac{\Pi}{\mu}$ and from second equation of system (3.1) we obtain $E_{H}=0$ and from third equation we get $E_{A}=0$ and from fourth equation we get $I=0$ and the last equation we get $R=0$.

Therefore the diseases free equilibrium point $Y_{0}$ is given by $Y_{0}=\left(\frac{\Pi}{\mu}, 0,0,0,0\right)$.

\subsection{The Endemic Equilibrium Point}

This is the situation where the disease persists, that is, where $I \neq 0, E_{H} \neq 0$ and $E_{A} \neq 0$ therefore the model has an endemic equilibrium point $Y_{1}$ given by. $Y_{1}=\left(S^{*}, E_{H}^{*}, E_{A}^{*}, I^{*}, R^{*}\right)$. Therefore, we solve system (3.1) for $S^{*}, E_{H}^{*}, E_{A}^{*}, I^{*}, R^{*}$. So from first equation of the system (3.1) we get

$$
Y_{1}=\left(S^{*}=\frac{\Pi}{\beta^{*}+\mu}\right)
$$

From second equation of the system (3.1) we get

$$
E_{H}^{*}=\frac{\prod \beta^{*} q(1-p)}{\left(\beta^{*}+\mu\right)(\mu+\tau)}
$$

From third equation of the system (3.1) we obtain

$$
E_{A}^{*}=\frac{\prod \beta^{*}(1-q)(1-p)}{\left(\beta^{*}+\mu\right)(\mu+\Upsilon)}
$$

Also from equation four of the system (3.1) we get

$$
I^{*}=\frac{\prod \beta^{*}((\mu+\Upsilon) \tau q(1-p)+(\mu+\tau) \Upsilon(1-q)(1-p)+(\mu+\tau)(\mu+\Upsilon))}{\left(\beta^{*}+\mu\right)(\mu+d+r)(\mu+\Upsilon)(\mu+\tau)}
$$


But $\beta^{*}=\frac{\alpha I}{N}$

Therefore

$$
I^{*}=\frac{\prod \alpha((\mu+\Upsilon) \tau q(1-p)+(\mu+\tau) \Upsilon(1-q)(1-p)+(\mu+\tau)(\mu+\Upsilon))-N \mu(\mu+d+r)(\mu+\Upsilon)(\mu+\tau)}{\alpha(\mu+d+r)(\mu+\Upsilon)(\mu+\tau)}
$$

Therefore

$$
I^{*}=\frac{\prod \alpha\left(C_{1}+C_{2}+C_{3}\right)-N \mu C_{4}}{\alpha C_{4}}
$$

where

$$
\begin{aligned}
& C_{1}=(\mu+\Upsilon) \tau q(1-p), \\
& C_{2}=(\mu+\tau) \Upsilon(1-q)(1-p), \\
& C_{3}=(\mu+\tau)(\mu+\Upsilon), \\
& C_{4}=(\mu+d+r)(\mu+\Upsilon)(\mu+\tau) .
\end{aligned}
$$

Consider fifth equation of the system (3.1) then we get.

$$
R^{*}=\frac{r \prod \beta^{*}((\mu+\Upsilon) \tau q(1-p)+(\mu+\tau) \Upsilon(1-q)(1-p)+(\mu+\tau)(\mu+\Upsilon))}{\mu\left(\beta^{*}+\mu\right)(\mu+d+r)(\mu+\Upsilon)(\mu+\tau)}
$$

Since $\beta^{*}=\frac{\alpha I^{*}}{N^{*}}$ therefore substituting Equation (3.15) in to $\beta^{*}=\frac{\alpha I^{*}}{N^{*}}$ then we obtain.

$$
\beta^{*}=\frac{\prod \alpha\left(C_{1}+C_{2}+C_{3}\right)-N^{*} \mu C_{4}}{N^{*} C_{4}}
$$

The case where $\beta^{*}=0$ results in the diseases free equilibrium is $E_{0}=\left(S^{*}, E_{H}^{*}, E_{A}^{*}, I^{*}, R^{*}\right)=\left(\frac{\Pi}{\mu}, 0,0,0,0\right)$.

The endemic equilibrium point is in terms of the force of infection, $\beta^{*}$.

Substituting $N^{*} \beta^{*}=\alpha I^{*}$ we get,

$$
I^{*}=\frac{\alpha N^{*}\left(A_{3}+A_{4}+b c\right)-a b c}{\alpha\left(A_{1}+A_{2}+A_{3}+A_{4}+b c+A_{5}+A_{6}+b a\right)}
$$

But $N^{*}=S^{*}+E_{H}^{*}+E_{\mathrm{A}}^{*}+I^{*}+R^{*}$

Therefore

$$
I^{*}=\frac{\alpha\left(S^{*}, E_{H}^{*}, E_{A}^{*}, I^{*}, R^{*}\right)\left(A_{3}+A_{4}+b c\right)-a b c}{\alpha\left(A_{1}+A_{2}+A_{3}+A_{4}+b c+A_{5}+A_{6}+b a\right)}
$$

where

$$
\begin{aligned}
& A_{1}=q(1-p) a c, \\
& A_{2}=(1-q)(1-p) b c, \\
& A_{3}=a \tau q(1-p), \\
& A_{4}=b \Upsilon(1-q)(1-p), \\
& A_{5}=\operatorname{ra\tau } q(1-p), \\
& A_{6}=b \Upsilon(1-q)(1-p) .
\end{aligned}
$$


After substituting the equilibrium point of in equation $\left(S^{*}, E_{H}^{*}, E_{A}^{*}, I^{*}, R^{*}\right)$ in Equation (3.18) we obtain.

$$
J_{1} \beta^{* 2}+J_{2} \beta^{*}-J_{3}=0
$$

where

$$
\begin{aligned}
& J_{1}=Z A \mu(\mu+\tau)(\mu+\Upsilon), \\
& J_{2}=\left(K_{1}-K_{2}-K_{3}-K_{4}-K_{5}\right), \\
& J_{3}=\alpha \pi \mu C_{4}(\mu+\tau)(\mu+\Upsilon) .
\end{aligned}
$$

where

$$
\begin{aligned}
& K_{1}=Z A \mu^{2}(\mu+\tau)(\mu+\Upsilon), \\
& K_{2}=\mu C_{4} q(1-p)(\mu+\tau), \\
& K_{3}=\mu C_{4}(1-q)(1-p)(\mu+\Upsilon), \\
& K_{4}=Z \mu C_{4}(\mu+\tau)(\mu+\Upsilon), \\
& K_{5}=r\left(C_{1}+C_{2}+C_{3}\right)(\mu+\tau)(\mu+\Upsilon) .
\end{aligned}
$$

Also

$$
\begin{aligned}
& Z=((\mu+\Upsilon) \tau q(1-p)+(\mu+\tau) \Upsilon(1-q)(1-p)+(\mu+\tau)(\mu+\Upsilon)), \\
& A=\alpha\left(A_{1}+A_{2}+A_{3}+A_{4}+b c+A_{5}+A_{6}+b a\right) .
\end{aligned}
$$

In which by using quadratic formula we get,

$$
\beta^{*}=\frac{-J_{2} \pm \sqrt{J_{2}^{2}+4 J_{1} J_{3}}}{2 J_{1}}
$$

This quadratic equation has one positive root when $\sqrt{J_{2}^{2}+4 J_{1} J_{3}}>J_{2}$, therefore, it shows that there exists at least one endemic equilibrium point.

\section{Numerical Simulations}

In this section, we carry out numerical simulations to illustrate analytical results obtained in the previous sections. We use a set of parameter values in the cited literature and estimate those which could not be found in literature to do the simulations of model (1), using Matlab software. Table 2 displays the set of parameter values to be used in the simulations and their sources.

\section{Simulations of Different Epidemiological Classes}

We simulated the parameters $q, p, \Upsilon$ and $\tau$ to check the contribution of these parameter and how they govern the animal TB transmission in overall TB transmissions.

The parameter varied above were hypothesized to contribute in the animal TB transmission, the parameters were varied individual and then were combined together to see the total contribution of the animal TB in overall TB.

Latent animal TB has high decrease compared to latent human TB, also Figure 2 indicates that as latent animal TB decrease then the overall number of susceptible individual decreases with higher rate that both latent animal and latent human TB (Figure 2).

In Figure 3 it shows that if we consider only human TB by varying $q$, only the susceptible class increase while when you combined both animal and human TB as in the Figure 4, susceptible class have fast decreasing this indicate that animal TB have major contribution in overall TB transmissions. The results are similar to results reported by (Mfinanga, et al., 2010).

In Figure 5, it indicates that animal TB transmission contribute to overall TB burden since when we vary $\Upsilon$ the exposed animal TB diminish and the infectious class increases and recovery individual also increase, Figure 6. The increase of recovery starts at year 0 - 5 and start to diminish slightly after year 5 . 
Table 2. Model parameters value.

\begin{tabular}{ccc}
\hline Parameters & Parameter Value & Source \\
\hline$\mu$ & 0.00987 per year & World Bank 2009 \\
$q$ & 0.083 year & Carlos Castillo-Chavez \\
$p$ & 0.35 per year & Estimated \\
$r$ & 0.85 per year & NTLP-Ministry of Health, Tanzania \\
$\tau$ & 0.0016 & Castillo Chavez and Song 2004 \\
$\beta$ & 0.0001 & Estimated \\
$d$ & 0.35 per year & Augusto, 2009 \\
& 0.00013 per year & US Global Heath Policy 2010
\end{tabular}

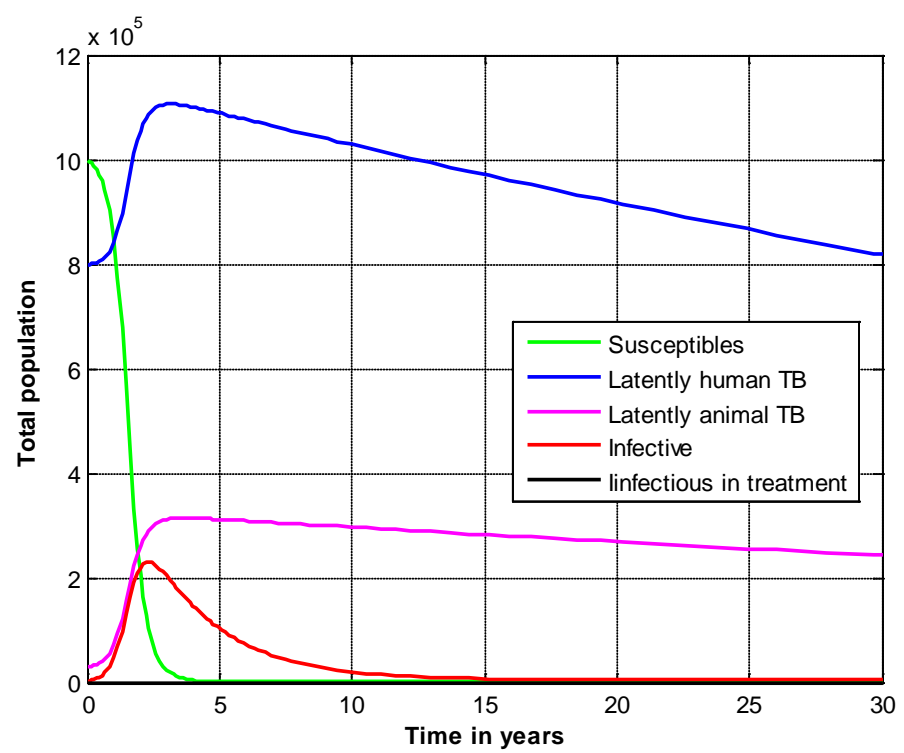

Figure 2. Illustration of total population to the time across five classes.

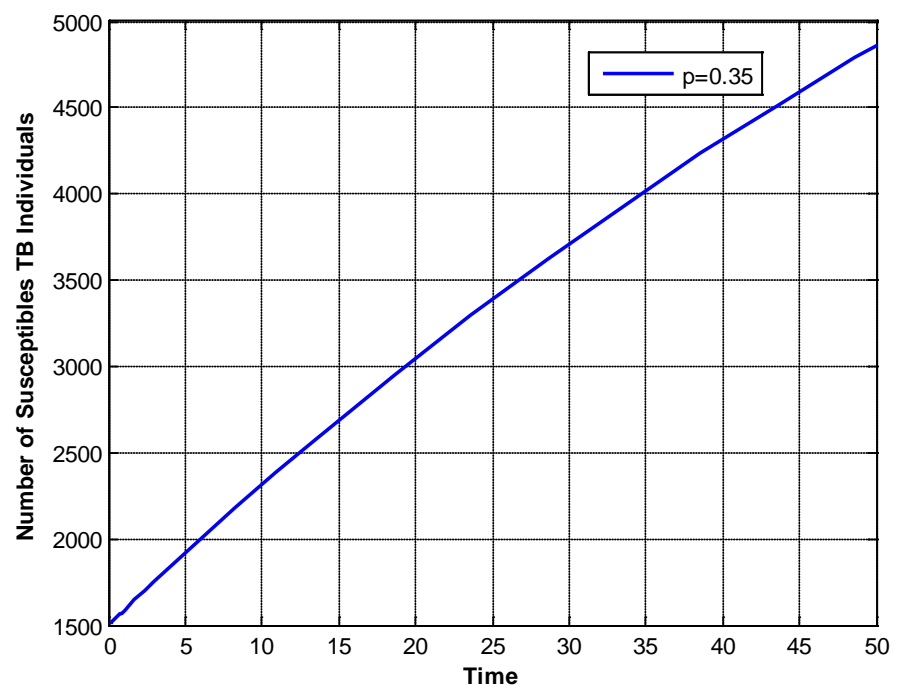

Figure 3. Susceptible class across human TB. 


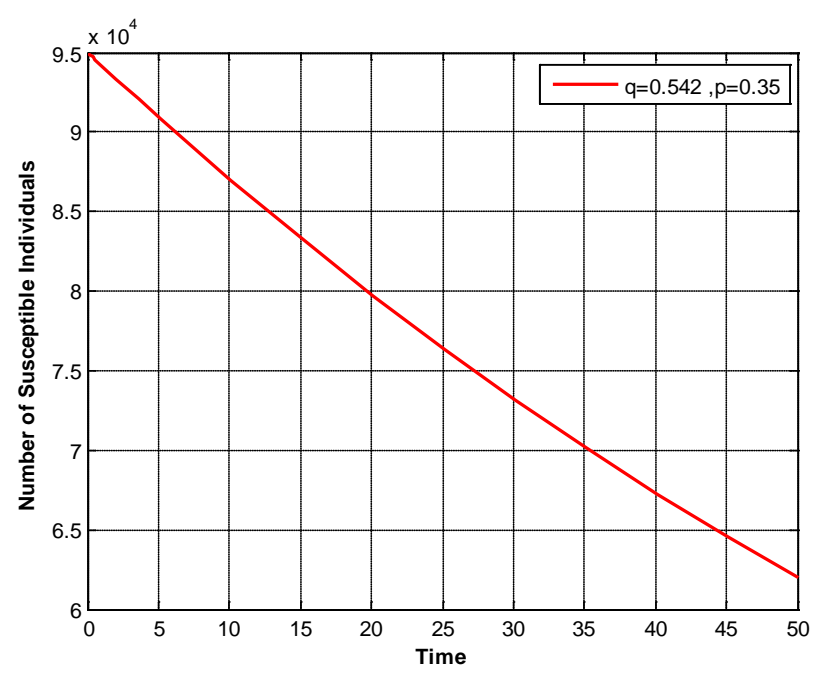

Figure 4. Susceptible class across years by varying both q-human TB and p-animal TB.

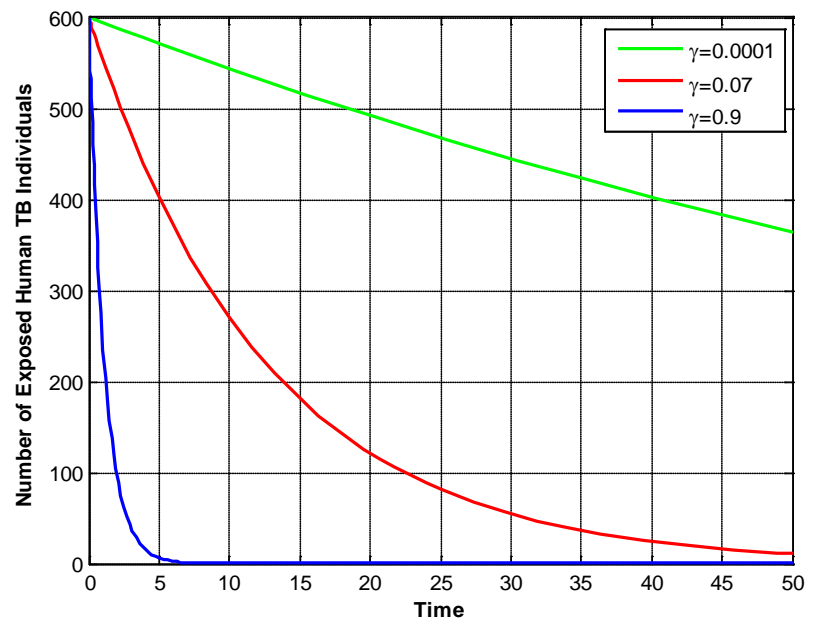

Figure 5. Exposed animal TB across animal TB transmission rate to active TB.

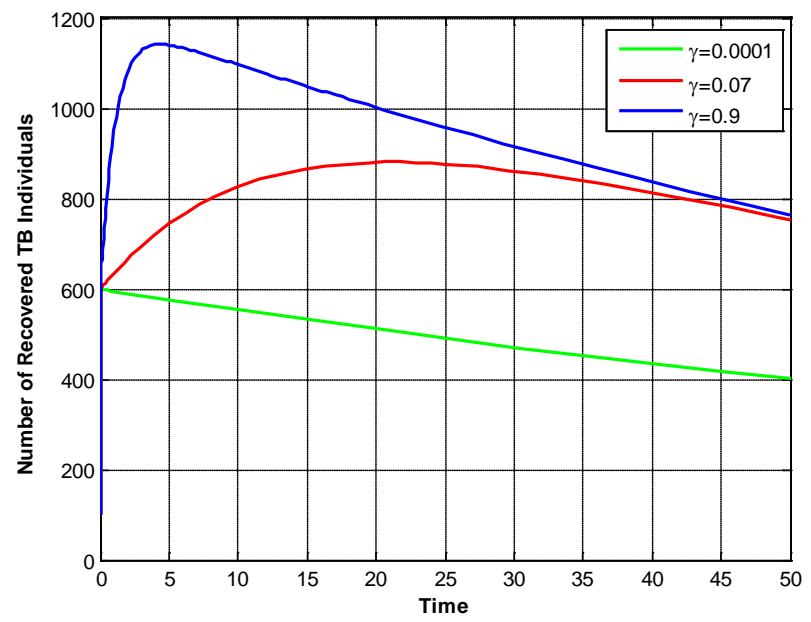

Figure 6. Recovery individual across change of $\Upsilon$-animal TB. 
Figure 7 above shows that as $\tau$ increases number of susceptible population decreases this is due to the fact that more exposed individual become infectious and then they are infecting other individuals in the population which lead to decrease in the number of susceptible population.

Figure 8 above shows that the higher the rate of infected individual to active TB, the lower the number of exposed TB in human class. This is due to the fact that those who are infected if they become active TB quickly they are being shifted to infectious class.

Figure 9 above indicates that the higher the rate TB transmissions rate in animal TB class the lower the number of exposed animal TB.

\section{Conclusion and Recommendation}

Transmission of TB from animal to human is a threaten to human being life as shown in the Equation (3.19). This clearly shows that human beings are at risk of being infected with TB from animals. Therefore, Ministry of Health and Social Welfare through NTLP with collaboration of Ministry of agriculture and livestock should ensure that beef/milk is clearly checked by veterinary officers.

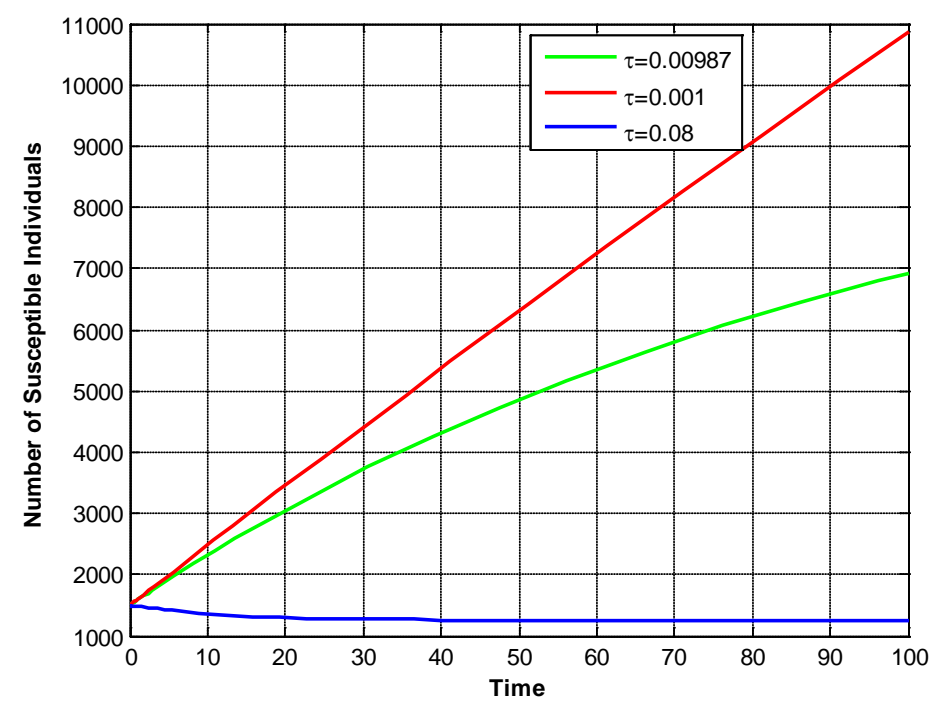

Figure 7. Susceptible class across transmission rate to infectious.

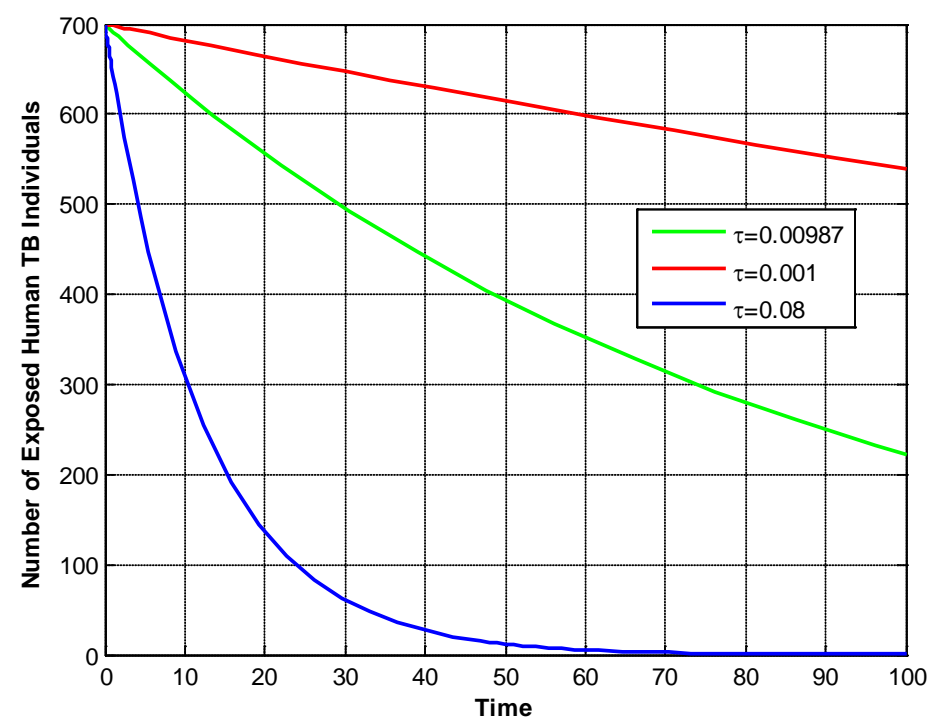

Figure 8. Illustration of change in exposed human TB in time. 


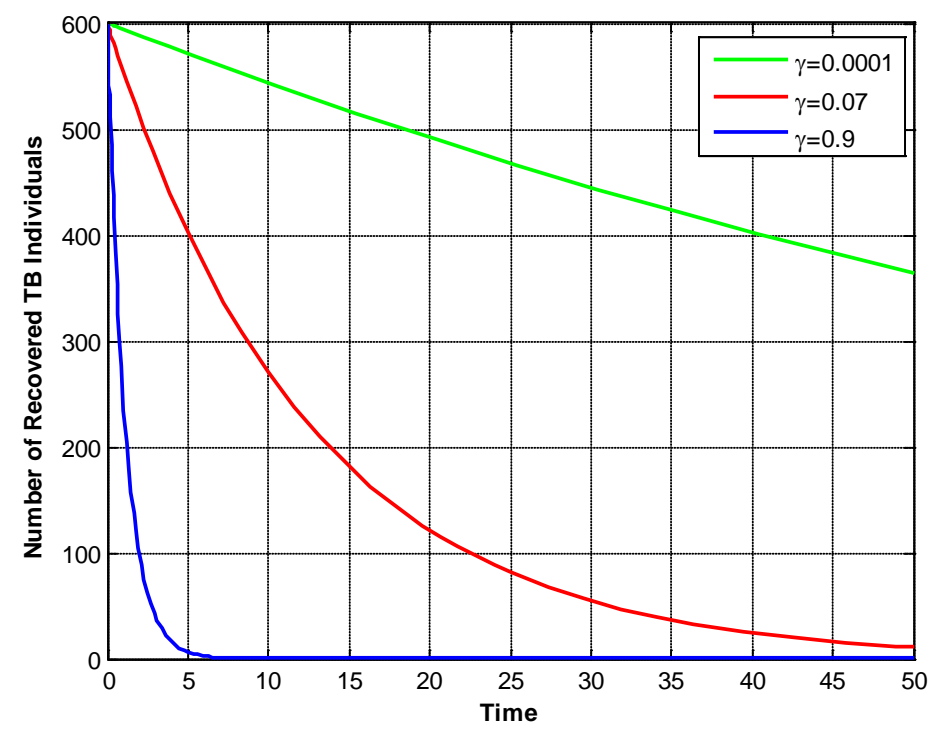

Figure 9. Illustration of change in exposed animal TB transferred to active TB.

\section{Acknowledgements}

First and foremost, I thank God for giving me ability, healthy and insight during preparation of this manuscript, secondly to Dr. Charles Mahera who coordinated the course to get the knowledge and skills to formulate the models, Third to Head of Mathematics Department at University of Dar es Salaam, Prof. Massawe for his kindness of giving us the nice place for learning.

\section{References}

[1] Adetunde, I.A. (2007) The Mathematical Models of the Dynamical Behavior of Tuberculosis Disease in the Upper East Region of the Northern Part of Ghana. A Case Study of Bawku. Research Journal of Applied Sciences, 2, 943-946.

[2] CDC (2005) Tuberculosis Cases and Percentages by Pulmonary and Extra pulmonary Diseases. US Department of Health and Human Services, Atlanta.

[3] World Health Organisation (1994) Zoonotic Tuberculosis (M. bovis): A Memoranda from WHO Meeting (with Participation of FAO). Bulletin of the WHO, 72, 851-857.

[4] Caffery, J.P. (1994) Studies of Bovine Tuberculosis Eradication Programmes in Europe. Veterinary Microbiology, 40, 1-4. http://dx.doi.org/10.1016/0378-1135(94)90041-8

[5] Shitaye, J.E., Tsegaye, W. and Pavlik, I. (2007) Bovine Tuberculosis Infection in Animal and Human Populations in Ethiopia: A Review. Veterinarni Medicina, 52, 317-332.

[6] WHO (1999) Global TB Program. TB Advocacy, a Practical Guide [Pamphlet]. WHO, Geneva.

[7] Beals, F.T. (2007) The Risk of Bovine Tuberculosis from Raw Milk Consumption with a Focus on Michigan in Wise Traditions in Food, Farming and the Healing Arts, the Quarterly Magazine of the Weston A. Price Foundation.

[8] Anaelom, N.J., Ikechukwu, O.J., Sunday, E.W. and Nnaemeka, U.C. (2010) Zoonotic Tuberculosis: A Review of Epidemiology, Clinical Presentation, Prevention and Control. Journal of Public Health and Epidemiology, 2, 118-124.

[9] Mfinanga, S.G., Mørkve, O., Kazwala, R.R., Cleaveland, S., Sharp, J.M., Shirima, G. and Nilsen, R. (2003) The Role of Livestock Keeping in Tuberculosis Trends in Arusha, Tanzania. International Journal of Tuberculosis and Lung Disease, 7, 695-704.

[10] Apariocio, J.P., Capurro, A.F. and Castillo-Chaveze, C. (2000) Transmission and Dynamics of Tuberculosis on Generalized Households. Journal of Theoretical Biology, 206, 327-341. http://dx.doi.org/10.1006/jtbi.2000.2129 
Scientific Research Publishing (SCIRP) is one of the largest Open Access journal publishers. It is currently publishing more than 200 open access, online, peer-reviewed journals covering a wide range of academic disciplines. SCIRP serves the worldwide academic communities and contributes to the progress and application of science with its publication.

Other selected journals from SCIRP are listed as below. Submit your manuscript to us via either submit@scirp.org or Online Submission Portal.
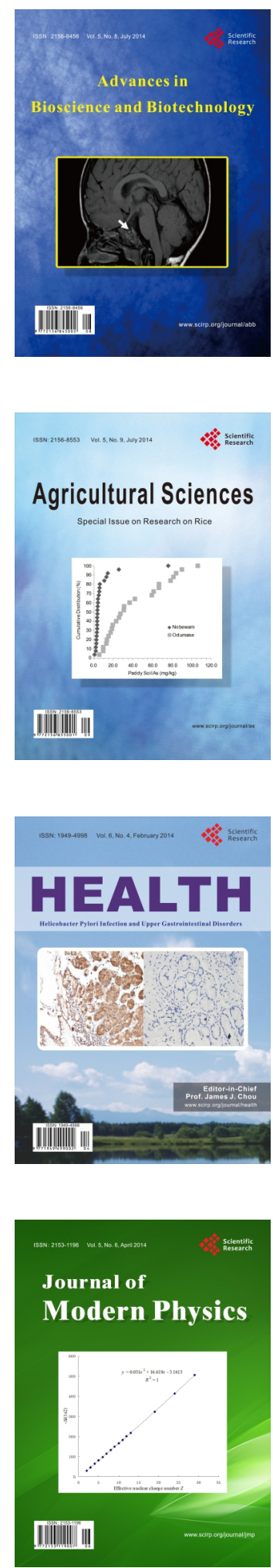
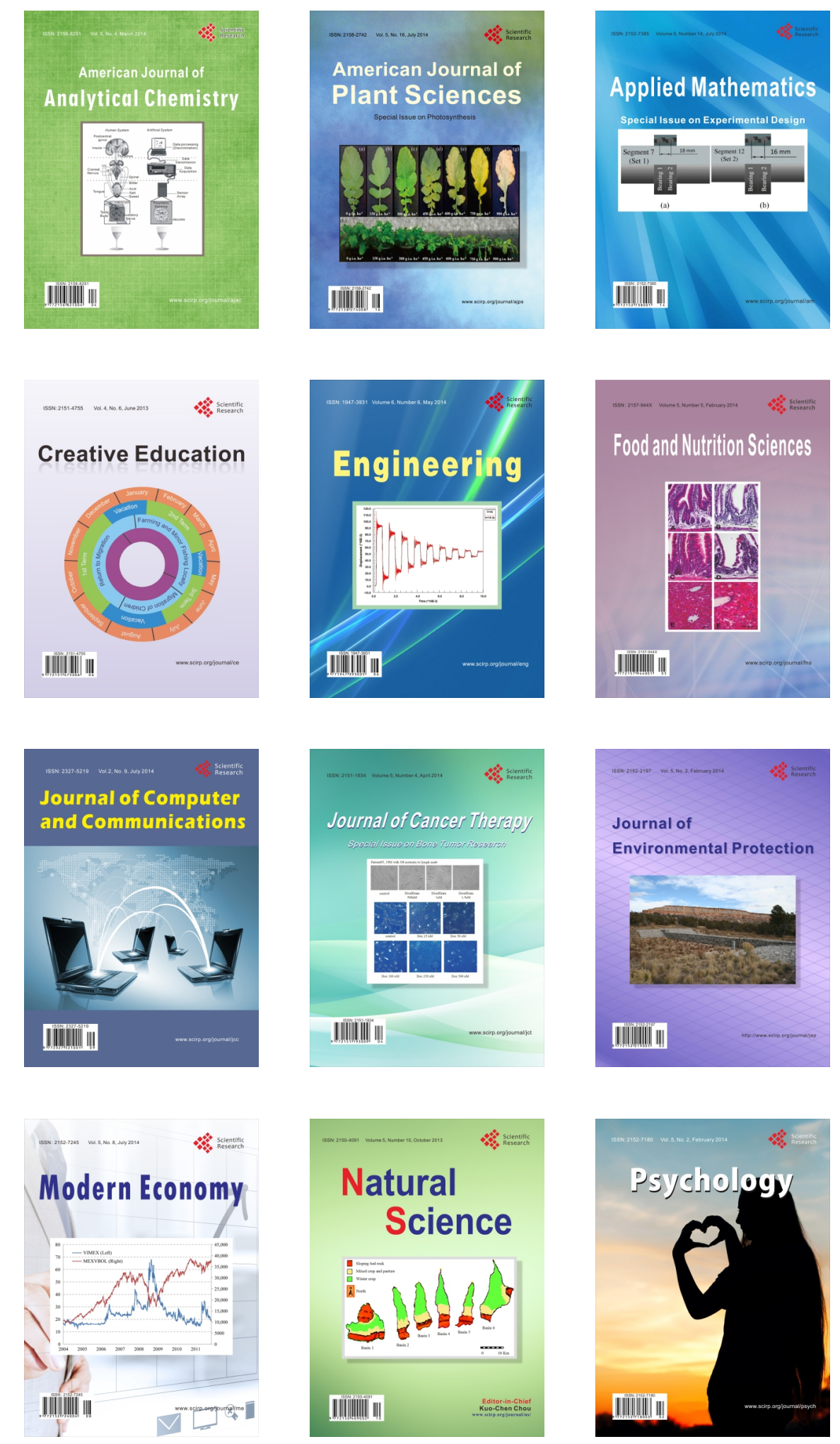